Ю. И. Черскиї (Олес. акад. слроительслиа и архияектуры)

\title{
ОБ АНАЛИТИЧНОСТИ ФУРЬЕ-ОРИГИНАЛА И ФУРЬЕ-ОБРАЗА ВНУТРИ ПІРОТИВОЛЕЖАЦИХ УГЛОВ
}

We prove that the Fourier image is an analytic function inside two alternate angles if the Fourier original possesses a similar property.

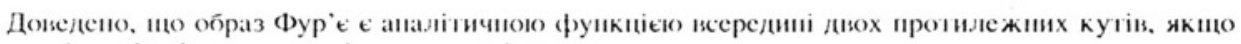

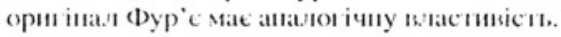

()динм из важных свойств образа Фурьс

$$
F(z)=\int_{-\infty}^{+\infty} f(t) e^{i z t} d t
$$

является ело аналитичность, если выполнены определенные условия, которые Так или иначе пейрализуют возможный рост экспоненты ехр (izt). До сих пор прн формулировке таких условнй исходили из представления $z=x+i y$, ко-

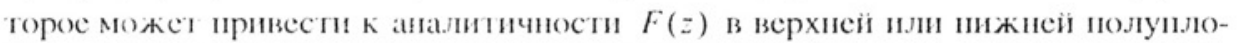
скоспи (нодоб̆о односторониему иреобразованио Ланласа), либо в горизон-

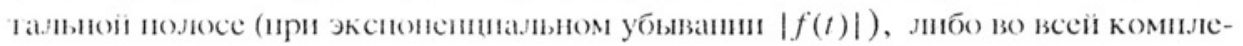
ксиои нлоскости (при спе болес быстром убывании $|f(t)|, t \rightarrow \pm \infty$ ).

Ниже пспользуется инос иредставление: $z=x \exp (i \varphi)$, гле $х и$ и $\varphi$ венеспвсни,. Для ораничениости модуля экспоненты $\exp (i z t)$ перемениую интегрирования $t$ следует взять в виде $t=s \exp (-i \varphi),-\infty<s<\infty$. При этом оригинал Фурье слелует состанпт, нз двух функциі, каждая из которых аналитична в одной из угловых областей:

$$
\begin{gathered}
\{\zeta \mid \arg \zeta \in(\alpha, \beta)\}, \\
\{\zeta \mid \arg (-\zeta) \in(\alpha, \beta)\} .
\end{gathered}
$$

Здесь и далысе венествениые числан $\alpha$ и $\beta$ таковы, что $-\pi \leq \alpha<0<\beta \leq \pi$ и $\beta$ $-\alpha \leq \pi$. Совокунноспь областей (2) и (3) обозначим через $D_{\alpha \beta}$ и будем говорн1ь. что функция $f(\zeta)$ аналитична в $D_{\alpha \beta}$.

В птоге вместо (1) получим следуюнце представление цля образа Фурье:

$$
F(z) \stackrel{\mathrm{d} f}{=} e^{-i \varphi} \int_{-\infty}^{+\infty} f\left(s e^{-i \varphi}\right) e^{i x s} d s, \quad z=x e^{i \varphi}
$$

Ниже доказано, что из аналитичности оригинала Фурье $f(\zeta)$ в $D_{\alpha \beta}$ следует аналитичность образа Фурье $F(z)$ в $D_{-\beta-\alpha}$.

$$
D_{-\beta-\alpha}=\{z \mid \arg z \in(-\beta,-\alpha)\} \cup\{z \mid \arg (-z) \in(-\beta,-\alpha)\} .
$$

\section{1. Формулировка теорем.}

Теорема 1. Пусть фулкция $f(\zeta)$ определена па вецествениої оси и аналиична в $D_{\alpha \beta}$. ПІусть при иелом числе $m \geq-3$ для лобово отрезка $\left[\alpha_{1}, \beta_{1}\right]$,

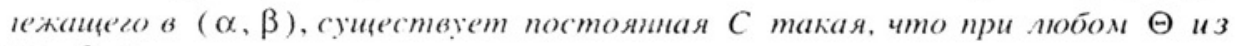
$\left\{\alpha_{1}, \beta_{1}\right\}$ 


$$
\begin{gathered}
\left|f\left(s e^{i \theta}\right)\right|<C(|s|+1)^{m}, \quad-\infty<s<\infty, \\
\left|\frac{d}{d s} f\left(s e^{i \theta}\right)\right|<C(|s|+1)^{m} \quad \text { npu } \quad s>0 \text { u } \quad s<0
\end{gathered}
$$

(функция $f(s)$ может ихеть разрыз в точке $\left.s^{\prime}=0\right)$. Тогда ее образ Фурье $F(z)$ аналитичен в $D_{-\beta-\alpha}$.

Доказательство опирается на утверждения следующей теоремы и дано в п. 3.

Теорема 2. Пусть функция $\psi(\zeta)$ определена и аналитична в угловой области (2), причем для любого отрезка $\left[\alpha_{1}, \beta_{1}\right] \subset(\alpha, \beta)$ суцествует постоянная $C$ такая, что при $s>0$ и $\Theta \in\left[\alpha_{1}, \beta_{1}\right]$

$$
\begin{gathered}
\left|\psi\left(s e^{i \theta}\right)\right|<C|s+1|^{-3} \\
\left|\frac{d}{d s} \psi\left(s e^{i \theta}\right)\right|<C|s+1|^{-3} .
\end{gathered}
$$

Kроме того, nусть

$$
\Psi(x, \varphi)=e^{-i \varphi} \int_{0}^{\infty} \Psi\left(s e^{-i \varphi}\right) e^{i x s} d s, \quad x \in \mathbb{R}^{1}, \quad-\beta<\varphi<-\alpha .
$$

Тогда равенство $\Psi(z)=\Psi(x, \varphi)$, где $z=x e^{i \varphi}$, определяет функцию $\Psi(z)$, аналитическую в $D_{-\beta-\alpha}$.

2. Доказательство теоремь 2. Пусть П - такой прямоугольник

$$
\Pi=\left\{(x, \varphi) \mid x_{0} \leq x \leq x_{1}, \quad \varphi_{0} \leq \varphi \leq \varphi_{1}\right\},
$$

что все точки $z=x e^{i \varphi}$ находятся в $D_{-\beta-\alpha}$. Докажем, что в прямоугольнике $\Pi$ функция $\Psi(x, \varphi)$ удовлетворяет уравненио Коши-Римана в полярных коорджнатах

$$
\frac{\partial \Psi(x, \varphi)}{\partial \varphi}=i x \frac{\partial \Psi(x, \varphi)}{\partial x} .
$$

Формула дифференцирования по $x$

$$
\frac{\partial \Psi(x, \varphi)}{\partial x}=i e^{-i \varphi} \int_{0}^{\infty} s e^{i x s} \psi\left(s e^{-i \varphi}\right) d s
$$

обоснована, например, в [1, с. 122].

Докажем равномерную непрерывность функции $\partial \Psi / \partial x$ по совокупности переменных $(x, \varphi)$ в П. Зададим $\varepsilon>0$ и пусть $(x, \varphi) \in \Pi,(\tilde{x}, \tilde{\varphi}) \in \Pi$. Используя неравенство (6), получаем

$$
\begin{gathered}
I_{1}=\left|\frac{\partial \Psi(x, \varphi)}{\partial x}-\frac{\partial \Psi(\tilde{x}, \varphi)}{\partial x}\right|= \\
=\left|\int_{0}^{\infty} s \Psi\left(s e^{-i \varphi}\right)\left[e^{i x s}-e^{i \tilde{x} s}\right] d s\right| \leq C \int_{0}^{\infty}\left|e^{i x x s}-e^{i \tilde{x} s}\right| \frac{d s}{s^{2}+1} .
\end{gathered}
$$

Фиксируем $N>0$ так, чтобы

$$
C \int_{N}^{\infty}\left|e^{i x s s}-e^{i \tilde{x} s}\right| \frac{d s}{s^{2}+1}<\frac{\varepsilon}{4}
$$


Тогда при $|x-\tilde{x}|<\delta_{1}$ и достаточно малом $\delta_{1}$ имесм

$$
I_{1} \leq \frac{\varepsilon}{4}+C \int_{0}^{N}\left|e^{i x s}-e^{i \tilde{x} s}\right| \frac{d s}{s^{2}+1}<\frac{\varepsilon}{2} .
$$

Осталось оценить модуль разности

$$
\begin{gathered}
I_{2}=\left|\frac{\partial \Psi(\tilde{x}, \varphi)}{\partial x}-\frac{\partial \Psi(\tilde{x}, \tilde{\varphi})}{\partial x}\right| \leq \\
\leq \int_{0}^{\infty}\left|s e^{-i \varphi} \psi\left(s e^{-i \varphi}\right)-s e^{-i \tilde{\varphi}} \psi\left(s e^{-i \tilde{\varphi}}\right)\right| d s=\int_{0}^{\sigma}+\int_{\sigma}^{N}+\int_{N}^{\infty} .
\end{gathered}
$$

Фиксируем числа $\sigma$ и $N$ так, чтобы интегралы с прсделами интегрирования 0 , $\sigma$ и $N, \infty$ не иревынали $\varepsilon / 6$. Опеним интеграл

$$
\int_{\sigma}^{N}\left|s e^{-i \varphi} \psi\left(s e^{-i \varphi}\right)-s e^{-i \bar{\varphi}} \psi\left(s e^{-i \bar{\varphi}}\right)\right| d s .
$$

На комиакес $\sigma \leq s \leq N, \varphi_{0} \leq \varphi \leq \varphi_{1}$ фуникия $\zeta \psi(\zeta), \zeta=s e^{i \varphi}$. в силу аналитичноти в каждой почке, иенрерьвна: согласио теореме Гейе [2] она и равномерно ненрерывна. ()скда следует сунествоване постоянной $\delta_{2}$ такой, чтони $|\varphi-\tilde{\varphi}|<\delta_{2}$ на компаке вынолияется нераненство

$$
\left|s e^{-i \varphi} \psi\left(s e^{-i \varphi}\right)-s e^{-i \bar{\varphi}} \psi\left(s e^{-i \bar{\varphi}}\right)\right|<\frac{\varepsilon}{6 N},
$$

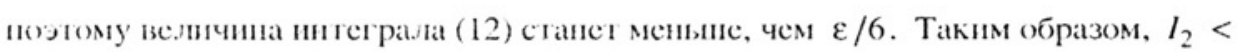
$<\varepsilon / 2$ и ири $|x-\tilde{x}|<\delta_{1}$ и $|\varphi-\tilde{\varphi}|<\delta_{2}$ сираведливо нераненство

$$
\left|\frac{\partial \Psi(x, \varphi)}{\partial x}-\frac{\partial \Psi(\tilde{x}, \tilde{\varphi})}{\partial x}\right| \leq I_{1}+I_{2}<\varepsilon .
$$

Раниомерная ненрерывность уетановлена.

Вонрос одиффференцировании но $\varphi$ функции $\Psi(x, \varphi)$ сводитсяк диффференцирозанию по параме тру $\varphi$ нитеграла

$$
\int_{0}^{\infty} \psi\left(s e^{-i \varphi}\right) e^{i x s} d s, \quad \varphi_{0} \leq \varphi \leq \varphi_{1} .
$$

где $x-$ фиксировано. Рассмотрим сначала интеграл

$$
\int_{0}^{1} \psi\left(s e^{-i \varphi}\right) e^{i x s} d s
$$

и покажем, что вынолиено известно [2, с. 381] условие, обеспечиваюиее дифрференцируемость интеграла по параметру, а именио - равномерная по совокунности неремениы s и $\varphi$ иенерывность производиой по $\varphi$ :

$$
\omega(s, \varphi)=-i s e^{-i \varphi} \psi^{\prime}\left(s e^{-i \varphi}\right) e^{i x s}
$$

в ирямоутолынике $0 \leq s \leq 1, \varphi_{0} \leq \varphi \leq \varphi_{1}$.

3ададим $\varepsilon>0$. Вследспвис (7) справедливо иеравенспво $|\omega(s, \varphi)| \leq C s$. Заклиочасм, что в нолосе $0 \leq s \leq \varepsilon / 4 C, \varphi_{0} \leq \varphi \leq \varphi_{1}$ модуль разности значений функции $\omega(s, \varphi)$ не превынает $\varepsilon / 2$ : 


$$
|\omega(s, \varphi)-\omega(\tilde{s}, \tilde{\varphi})| \leq \frac{\varepsilon}{2} .
$$

В оставшемся прямоугольнике

$$
\frac{\varepsilon}{4 C} \leq s \leq 1, \quad \varphi_{0} \leq \varphi \leq \varphi_{1}
$$

функция $\omega(s, \varphi) \exp (-i x s)=-i \zeta \psi^{\prime}(\zeta)$ согласно теореме Гейне равномерно непрерывна по совокупности $(s, \varphi)$; вместе с ней будет равномсрно непрерывна в прямоугольнике (15) и функцня $\omega(s, \varphi)$. Значнт, существует число $\delta>0$ такое, что если $|s-\tilde{s}|<\delta$ и $|\varphi-\tilde{\varphi}|<\delta$, где $s, \tilde{s}, \varphi, \tilde{\varphi}$ взяты из прямоугольника (15), то

$$
|\omega(s, \varphi)-\omega(\tilde{s}, \tilde{\varphi})| \leq \frac{\varepsilon}{2}
$$

Сопоставляя это неравенство с (14), внднм, что в прямоугольнике $0 \leq s \leq 1$, $\varphi_{0} \leq \varphi \leq \varphi_{1}$ при $|s-\tilde{s}|<\delta$ и $|\varphi-\tilde{\varphi}|<\delta$ справедлнво неравенство

$$
|\omega(s, \varphi)-\omega(\tilde{s}, \tilde{\varphi})|<\varepsilon,
$$

что и означает равномернуго непрерывность. Итак,

$$
\frac{\partial}{\partial \varphi} \int_{0}^{1} \psi\left(s e^{-i \varphi}\right) e^{i x s} d s=-i e^{-i \varphi} \int_{0}^{1} s \psi^{\prime}\left(s e^{-i \varphi}\right) e^{i . x s} d s
$$

Для интеграла

$$
\int_{1}^{\infty} \psi\left(s e^{-i \varphi}\right) e^{i \cdot x s} d s, \quad \varphi_{0} \leq \varphi \leq \varphi_{1},
$$

провернм выполнение условнй теоремы [2, с. 486] о диф)еренцированни несобственного интеграла. Действнтельно, во-первых, ннтеграл (17) сходится, что сліедует из предположения (6). Во-вторых, функция (13) равномерно непрерывна по совокупности переменных $s$ н $\varphi$ в прямоугольнике (при любом $h>0$ ): $1 \leq s \leq 1+h, \varphi_{0} \leq \varphi \leq \varphi_{1}$. В-третьн, несобственный ннтеграл

$$
\int_{1}^{\infty} \frac{\partial}{\partial \varphi} \psi\left(s e^{-i \varphi}\right) e^{i x s} d s=-i \int_{1}^{\infty} e^{-i \varphi} s \psi^{\prime}\left(s e^{-i \varphi}\right) e^{i x s} d s
$$

равномерно сходится по $\varphi \in\left[\varphi_{0}, \varphi_{1}\right]$, что следует из предположення (7). Согласно указанной теореме допустимо дифференцирование по $\varphi$ под знаком ннтеграла (17), которое вместе с равенством (16) приводит к результату

$$
\begin{gathered}
\frac{\partial \Psi(x, \varphi)}{\partial \varphi}=-i e^{-i \varphi} \int_{0}^{\infty} \psi\left(s e^{-i \varphi}\right) e^{i . x s} d s- \\
-i e^{-2 i \varphi} \int_{0}^{\infty} s \psi^{\prime}\left(s e^{-i \varphi}\right) e^{i x s} d s, \quad(x, \varphi) \in \Pi .
\end{gathered}
$$

Провернм выполненне условня Коши-Рнмана. Покажем, что тождество (10) имеет место в прямоугольнике (9). С помощыо равенств (11) и (18) прнводим тождество (10) к равносильному тождеству 


$$
\int_{0}^{\infty} s e^{i x s} \frac{\partial}{\partial s} \psi\left(s e^{-i \varphi}\right) d s=-\int_{0}^{\infty} \psi\left(s e^{-i \varphi}\right) \frac{\partial}{\partial s}\left(s e^{i x s}\right) d s .
$$

Одиако последнес тождество - формула интегрирования по частям, причем вниолиена условия ес ирименимости [2, с. 467]. В частности, сунествуют пре-

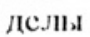

$$
\lim _{s \rightarrow 0} s e^{i x s} \psi\left(s e^{-i \varphi}\right)=0, \quad \lim _{s \rightarrow \infty} s e^{i x s} \psi\left(s e^{-i \varphi}\right)=0 .
$$

Поскольку уравнение Кони-Римана тождественио вынолияется в ирямоугольнкс П. тов П пронзводная $\partial \Psi / \partial \varphi$ будет пенрерывной вследствие неирерњљносп функции $\partial \Psi / \partial x$, а функция $\Psi(z)=\Psi(x, \varphi)-$ аналитической

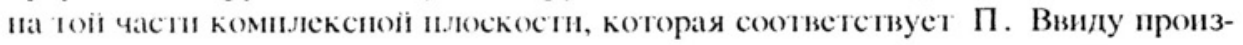
вольности выорора П фуликия $\Psi(z)$ будет аналитическої в $D_{-\beta-\alpha}$. Теорема 2 доказанtа.

\section{3. Доказате.истио теоремы 1. Функции}

$$
\begin{gathered}
\psi(\zeta)=f(\zeta)(\zeta+1)^{-3-m}, \quad \arg \zeta \in(\alpha, \beta), \\
\psi_{1}(\zeta)=f(-\zeta)(-\zeta-1)^{-3-m}, \quad \arg \zeta \in(\alpha, \beta) .
\end{gathered}
$$

удовленворяют весм условням георемы 2, так что их Фурыс-образы $\Psi(z)$ и $\Psi_{1}(z)$ аналипичны в $D_{-\beta-\alpha}$. Bыразим Фурье-оригинал $f(x)$ через $\psi(x)$ и

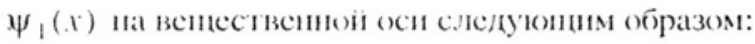

$$
f(x)=\frac{1+\operatorname{sgn} x}{2} \psi(x)(x+1)^{3+m}+\frac{1-\operatorname{sgn} x}{2} \psi_{1}(-x)(x-1)^{3+m} .
$$

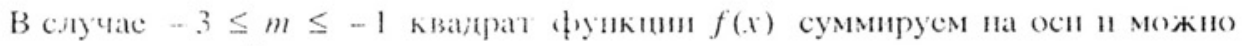

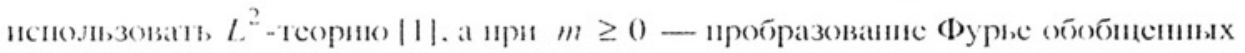

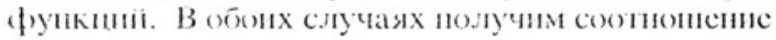

$$
F(x)=\left(-i \frac{d}{d x}+1\right)^{3+m} \Psi(x)+\left(-i \frac{d}{d x}-1\right)^{3+m} \Psi_{1}(-x) .
$$

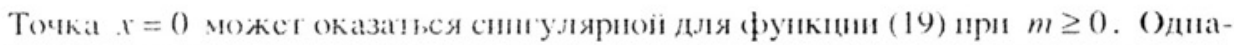
ко энобудет единственая синулярная точка. Вес осталыныс точки веныествениои оси как в случас $-3 \leq m \leq-1$, так и нри $m \geq 0$ - точки аналитичноспи фулкини (19). Болес того. Тождество (19) позволяет аналитически продол-

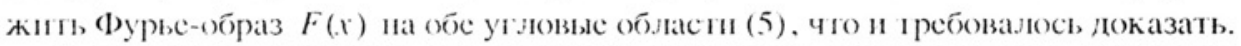

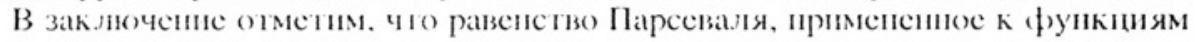
из формулы (4), даст онределениы количествениые соонопения между ори-

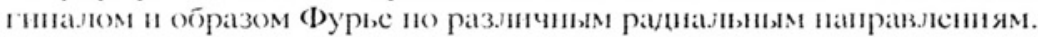

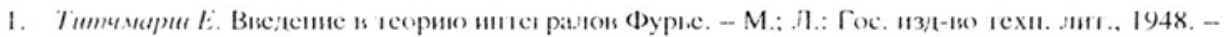
$479 \mathrm{c}$.

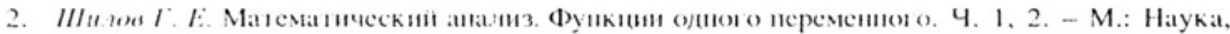
$1969 .-528 \mathrm{c}$. 